PROCEEDINGS OF THE

AMERICAN MATHEMATICAL SOCIETY

Volume 135, Number 8, August 2007, Pages 2629-2631

S 0002-9939(07)08696-0

Article electronically published on February 2, 2007

\title{
ON FUNDAMENTAL GROUPS OF COMPACT HAUSDORFF SPACES
}

\author{
JAMES E. KEESLING AND YULI B. RUDYAK
}

(Communicated by Alexander N. Dranishnikov)

\begin{abstract}
We discuss which groups can be realized as the fundamental groups of compact Hausdorff spaces. In particular, we prove that the claim "every group can be realized as the fundamental group of a compact Hausdorff space" is consistent with the Zermelo-Fraenkel-Choice set theory.
\end{abstract}

In this note we prove that every group $\pi$ can be realized as the fundamental group of a compact Hausdorff space, if we work in the pattern of the set theory without inaccessible cardinals. (See e.g. 2] concerning inaccessible cardinals.) Note that, because of a theorem of Kuratowski [2, Proposition 1.2], the absence of inaccessible cardinals is consistent with the Zermelo-Fraenkel-Choice set theory.

The idea is the following. Take a $C W$-space $X$ with $\pi_{1}(X)=\pi$ and let $\beta X$ be the Stone-Čech compactification of $X$, 4. Then $X$ is a path-connected component of $\beta X$, and therefore $\pi_{1}(\beta X, *)=\pi$ for all $* \in X$.

This result should be contrasted with the result of Saharon Shelah [5] that for path connected, locally path connected compact metric spaces $X, \pi_{1}(X)$ is either a finitely generated group or has cardinality $2^{\aleph_{0}}$.

We are grateful to Alex Dranishnikov for useful discussions. We must also mention that this paper was initiated and stimulated by the Algebraic Topology Discussion List.

All spaces are assumed to be Hausdorff, all maps and functions are assumed to be continuous. We denote by $I$ the unit segment $[0,1]$. Let $\mathbb{N}^{*}$ denote the one-point compactification of the natural numbers $\mathbb{N}$ with $*$ being the point at infinity.

A dated, but useful, compendium of information on the Stone-Čech compactification can be found in [6]. See also [1]. The Stone-Čech compactification $\beta X$ of the completely regular space $X$ can be characterized as a topological embedding $X \subset \beta X$ with $\beta X$ compact and such that $X$ is dense in $\beta X$ and such that every function $f: X \rightarrow I$ can be extended to a function $\widehat{f}: \beta X \rightarrow I$. There are several standard constructions of the Stone-Čech compactification. One construction uses the maximal ideals in the ring of bounded functions on $X, C^{*}(X)$, with the hull-kernel topology [1, Chapter 7].

Let $v X$ denote the Hewitt realcompactification of $X$, [1, Chapter 8]. Recall that $X \subset v X \subset \beta X$ and is characterized by the property that every continuous function

Received by the editors April 19, 2005 and, in revised form, March 3, 2006.

2000 Mathematics Subject Classification. Primary 55Q05; Secondary 03E10, 03E55, 54C30, 54D30, 54D60.

The second author was supported by NSF grant 0406311.

(C)2007 American Mathematical Society 
$X \rightarrow \mathbb{R}$ (not necessarily bounded) can be extended to $v X$. A space $X$ is called realcompact if $v X=X$. Note that a realcompact space is not necessarily compact.

1. Theorem. If $X$ is a paracompact space of non-measurable cardinality, then $v X=X$.

Proof. Katetov 3 proved that a paracompact space $X$ is realcompact iff each of its closed discrete subspaces is realcompact. On the other hand, a discrete subspace is realcompact iff it has non-measurable cardinality, 11, Chapter 12].

2. Theorem. Each non-discrete, closed subset in $\beta X \backslash v X$ contains a copy of $\beta \mathbb{N}$, and so its cardinality is at least $2^{\mathrm{c}}$.

Proof. See [1, Theorem 9.11].

3. Theorem. If $X$ is a path connected paracompact space of non-measurable cardinality, then $X$ is a path component of $\beta X$.

Proof. Suppose that there exists a path $\alpha: I \rightarrow \beta X$ with $\alpha(0) \in X$ and $\alpha(1) \in$ $\beta X \backslash X$. Then, by Theorems 1 and 2$] \alpha(I) \cap(\beta X \backslash X)$ is a discrete set. So, we may assume without loss of generality that $\alpha([0,1)) \subset X$ and $\alpha(1) \in \beta(X) \backslash X$. Clearly, then $\alpha([0,1))$ is an infinite set; otherwise $\alpha(1) \in X$.

Now let $\left\{t_{n}\right\}_{n=1}^{\infty}$ be a sequence of distinct points in $\alpha([0,1))$ converging to $\alpha(1)$. Define $f\left(t_{n}\right)=n \bmod 2:\left\{t_{n}\right\}_{n=1}^{\infty} \rightarrow[0,1]$. Since $X$ is paracompact and therefore normal, let $F: X \rightarrow[0,1]$ be any extension of $f$ to all of $X$ using Tietze's Extension Theorem. Then let $\widehat{F}: \beta X \rightarrow[0,1]$ be the Stone-Čech extension of $F$ to $\beta X$. Then $\widehat{F} \mid\left\{t_{n}\right\}_{n=1}^{\infty}=f$ has an extension to $\left\{t_{n}\right\}_{n=1}^{\infty} \cup \alpha(1)$, which is clearly a contradiction.

4. Corollary. Every group of non-measurable cardinality is the fundamental group of a compact space.

Proof. Let $\pi$ be a given group. Let $X$ be a connected $C W$-space having $\pi_{1}(X)=\pi$. We can assume that $X$ has non-measurable cardinality. Since $X$ is paracompact, we conclude that $\pi_{1}\left(\beta X, x_{0}\right)=\pi$ for any $x_{0} \in X$ in view of Theorem 3 .

5. Remark. Similar arguments show that, given a sequence $\left\{A_{n}\right\}_{n=1}^{\infty}$ of groups of non-measurable cardinality, with $A_{n}$ abelian for $n \geq 2$, there exists a compact space $X$ with $\pi_{n}(X)=A_{n}$ for all $n \geq 1$.

6. Remark. Let us recall that every measurable cardinal is inaccessible, 2].

7. Remark. Of course, there exist groups of measurable cardinality that can be realized as the fundamental groups of compact spaces. For example, the product of any family of circles is compact, and so $\mathbb{Z}^{m}$ with any cardinal $m$ can be realized.

8. Remark. In the first version of the paper (that we put in an electronic archive) we asked whether there exists a path connected compact space with prescribed fundamental group of non-measurable cardinality. Recently Adam Przeździecki notified us that the answer is affirmative. Also, he announced an example of a group of measurable cardinality that is not the fundamental group of any compact space. 


\section{REFERENCES}

[1] L. Gillman and M. Jerison, Rings of Continuous Functions, Springer-Verlag, 1976 (reprint of the 1960 edition). MR0407579(53:11352)

[2] A. Kanamori, The Higher Infinite, Springer-Verlag, 1997. MR.1321144 (96k:03125)

[3] M. Katětov, Measures in fully normal spaces. Fund. Math. 38 (1951), 73-84. MR0048531 $(14: 27 \mathrm{c})$

[4] J. Munkres, Topology, Second Edition, Prentice-Hall, 2000. MR 0464128 (57:4063)

[5] S. Shelah, Can the fundamental (homotopy) group of a space be the rationals? Proc. Amer. Math. Soc. 103 (1988), 627-632. MR0943095 (89g:55021)

[6] R. Walker, The Stone-Čech Compactification, Springer-Verlag, 1974. MR0380698 (52:1595)

Department of Mathematics, University of Florida, 358 Little Hall, Gainesville, FLORIDA 32611-8105

E-mail address: jek@math.ufl.edu

Department of Mathematics, University of Florida, 358 Little Hall, Gainesville, FLORIDA 32611-8105

E-mail address: rudyak@math.ufl.edu 